\title{
Hospital volume-outcome relationship in severe traumatic brain injury: stratified analysis by level of trauma center
}

\author{
*Sung Huang Laurent Tsai, MD, MPH,,-3 Anshit Goyal, MBBS, ${ }^{1,4}$ Mohammed Ali Alvi, MBBS, $, 2,4$ \\ Panagiotis Kerezoudis, MD, MS, ${ }^{2,4}$ Yagiz Ugur Yolcu, MD, ${ }^{2,4}$ Waseem Wahood, MS, ${ }^{2,4}$ \\ Elizabeth B. Habermann, PhD, ${ }^{5}$ Terry C. Burns, MD, PhD, ${ }^{4}$ and Mohamad Bydon, MD $^{2,4}$ \\ ${ }^{1}$ Johns Hopkins Bloomberg School of Public Health, Baltimore, Maryland; ${ }^{2}$ Mayo Clinic Neuro-Informatics Laboratory, \\ Mayo Clinic; Departments of ${ }^{4}$ Neurologic Surgery and ${ }^{5}$ Health Sciences Research, Mayo Clinic, Rochester, Minnesota; and \\ ${ }^{3}$ Department of Orthopedic Surgery, Chang Gung Memorial Hospital, Keelung, Taiwan
}

\begin{abstract}
OBJECTIVE The nature of the volume-outcome relationship in cases with severe traumatic brain injury (TBI) remains unclear, with considerable interhospital variation in patient outcomes. The objective of this study was to understand the state of the volume-outcome relationship at different levels of trauma centers in the United States.

METHODS The authors queried the National Trauma Data Bank for the years 2007-2014 for patients with severe TBI. Case volumes for each level of trauma center organized into quintiles (Q1-Q5) served as the primary predictor. Analyzed outcomes included in-hospital mortality, total hospital length of stay (LOS), and intensive care unit (ICU) stay. Multivariable regression models were performed for in-hospital mortality, overall complications, and total hospital and ICU LOSs to adjust for possible confounders. The analysis was stratified by level designation of the trauma center. Statistical significance was established at $p<0.001$ to avoid a type I error due to a large sample size.

RESULTS A total of 122,445 patients were included. Adjusted analysis did not demonstrate a significant relationship between increasing hospital volume of severe TBI cases and in-hospital mortality, complications, and nonhome hospital discharge disposition among level I-IV trauma centers. However, among level II trauma centers, hospital LOS was longer for the highest volume quintile (adjusted mean difference [MD] for Q5: 2.83 days, $95 \% \mathrm{Cl} 1.40-4.26$ days, $p<0.001$, reference = Q1). For level III and IV trauma centers, both hospital LOS and ICU LOS were longer for the highest volume quintile (adjusted MD for Q5: LOS 4.6 days, 95\% Cl 2.3-7.0 days, $p<0.001$; ICU LOS 3.2 days, 95\% Cl 1.6-4.8 days, $p$ $<0.001)$.

CONCLUSIONS Higher volumes of severe TBI cases at a lower level of trauma center may be associated with a longer LOS. These results may assist policymakers with target interventions for resource allocation and point to the need for careful prehospital decision-making in patients with severe TBI.

https://thejns.org/doi/abs/10.3171/2020.1.JNS192115
\end{abstract}

KEYWORDS traumatic brain injury; head trauma; surgery; hospital volume; outcome analysis; volume-outcome relationship; National Trauma Data Bank; mortality; length of stay; ICU days

$\mathrm{T}$ HE volume-outcome relationship in trauma centers has been a subject of debate in the last 2 decades. Conflicting analyses have demonstrated that a higher case volume is associated with improved outcomes $5,10,12,14,16,22$ or is not associated with any overall effect. $3,7,9,11,17$ The American College of Surgeons (ACS) has developed guidelines outlining minimum volume standards for level I and level II trauma centers. ${ }^{2}$ For example, according to the ACS, level I trauma centers are required to admit at least 1200 trauma patients yearly or to have 240 admissions with an Injury Severity Score (ISS) of more than 15. Larger volumes of patients provide the trauma team with more experience that should result in improved outcomes. ${ }^{15}$ A minimum volume is needed for experience, but at a certain point, overloading trauma center resources with minimally injured patients may diminish their effectiveness. ${ }^{13}$

Although many surgical subspecialties have demon-

* S.H.L.T. and A.G. have contributed equally to this work. 
strated the volume-outcome relationship among several elective procedures, such as endocrine, vascular, cardiac, gastrointestinal, and urological procedures, the nature of this relationship in traumatic brain injury (TBI) cases remains unclear, with considerable interhospital variation in patient outcomes. ${ }^{5,7,9,10,12,14,16-18,22}$ Some studies have demonstrated no significant relationship, while others have found a significant impact of hospital volume on outcomes in TBI. ${ }^{1,21}$ Inconsistent results from these studies may be attributable to differing statistical analyses, source populations, databases, or geographic variations.,20,21 To address this knowledge gap, we queried a national trauma registry to understand the state of the volume-outcome relationship at different levels of trauma centers in the United States.

\section{Methods \\ Data Source and Patient Population}

This cohort study was conducted to analyze the hospital volume-outcome relationship in TBI. We queried the National Trauma Data Bank (NTDB) for the years 20072014 for adult patients (age $\geq 18$ years) with an admission diagnosis of severe TBI, defined as Glasgow Coma Scale (GCS) score $\leq 8$, and an Abbreviated Injury Scale (AIS) head score $\geq 3$. We excluded nonsurvival TBI with an AIS head score of 6 , dead on arrival, and penetrating TBI. The NTDB contains information on hospital characteristics, patient demographics, prehospital care, diagnoses, injury severity scoring, inpatient care, complications, as well as outcomes. The NTDB is the largest aggregation of trauma registry data ever assembled.

\section{Variables}

The primary predictor was defined as the annual volume (organized into quintiles: Q1-Q5) of severe TBI cases at the hospital (calculated separately for each designated level of trauma center). Patient-level covariates of interest included demographics such as age, sex, and race; insurance status; comorbidities; type of surgical intervention (burr hole, craniectomy, and removal of hematoma); type of intracranial pathology (epidural, subdural, brainstem, and cerebellar hematomas); GCS score; hypotension on admission (defined as systolic blood pressure < $90 \mathrm{~mm} \mathrm{Hg}$ ); ISS score; and complications. The primary outcome evaluated was in-hospital mortality. Secondary outcomes included 1) overall complications (acute kidney injury, acute respiratory distress syndrome, cardiac arrest, catheter-associated urinary tract infection, central line-associated bloodstream infection, deep surgical site infection, deep vein thrombosis, pulmonary embolism, myocardial infarction, organ/space surgical site infection, pressure ulcer, severe sepsis, stroke, superficial incisional surgical site infection, unplanned admission to the intensive care unit [ICU], ventilator-associated pneumonia, or unplanned return to the operating room), 2) total hospital length of stay (LOS), 3) ICU stay, and 4) nonhome discharge. The ICD-9-CM procedural codes for adjusting the outcome included craniectomy $(01.23,01.25,01.26,01.39$, 01.51, and 02.01), burr hole (01.09), removal of epidural hematoma (01.24), removal of subdural hematoma (01.31), removal of intracerebral hematoma (01.39), and intracranial pressure (ICP) monitoring (01.10). The type of intracranial pathology was defined using ICD-9-CM diagnosis codes: epidural hematoma (852.4), subdural hematoma (852.2 and 852.3), and intracerebral lesions (851.4, 851.5, 851.6, 851.7, 852.0, and 852.1).

\section{Statistical Analysis}

All statistical analyses were performed using Stata/MP statistical software version 14.2 (1985-2015, StataCorp LLC). Descriptive statistics were computed for patientlevel and hospital-level characteristics. Categorical and continuous variables were compared using chi-square and two-sample t-tests. A multilevel mixed-effects logistic regression model was used to evaluate in-hospital mortality. Similarly, a mixed-effects linear regression was fitted for total hospital LOS and ICU stay (days) with hospital volume of severe TBI cases as the primary predictor. To account for hospital-level covariates, hospital characteristics such as number of beds and teaching status were added as covariates, while the results were clustered for reporting facility. Some authors have suggested that multilevel modeling should be considered to evaluate in-hospital mortality in trauma patients. ${ }^{4}$ Both regression models were adjusted for the following variables: age, sex, race, insurance, hypotension on admission (systolic blood pressure < $90 \mathrm{~mm} \mathrm{Hg}$ ), ISS score, preadmission interhospital transfer, GCS score on admission, comorbidities (hypertension, diabetes, cerebrovascular accident, hemodialysis, and cancer with chemotherapy), intracranial lesions (epidural, subdural, brainstem, and cerebellar hematomas), utilization of ICP monitoring, and procedure performed (burr hole and craniectomy). Adjustments were based on the guidelines reported in the article by Haider et al. ${ }^{8}$ The analysis was stratified by level of trauma center. Adjusted odds ratios and coefficients with $95 \%$ confidence intervals were calculated from the logistic regression analysis. For LOS, the outcome of interest was the adjusted mean difference (MD; in days) along with $95 \%$ confidence intervals. The $p$ values were two-sided, and statistical significance was established at $\mathrm{p} \leq 0.001$ (because of the large sample size).

\section{Results \\ Study Population}

Between 2007 and 2014, a total of 5,836,508 patients were registered in the NTDB data set. After applying our inclusion and exclusion criteria, 77,592 subjects with severe TBI were eligible for analysis. Among level I trauma centers, low- to high-volume quintiles were identified as $\leq 357,358-482,483-642,643-765$, and $\geq 766$ cases of severe TBI annually. For level II trauma centers, volume quintiles of severe TBI (low to high) were identified as $\leq 121,122-189,190-240,241-322$, and $\geq 323$ cases/year. Tables 1 and 2 summarize the patient-level and hospitallevel characteristics of level I and level II trauma centers by volume. The proportion of the Hispanic population showed an increasing trend from low- to high-volume hospitals $(10.8 \%$ to $14.7 \%)$. As for the GCS score, however, more severe cases (GCS 3) appeared in higher-volume 


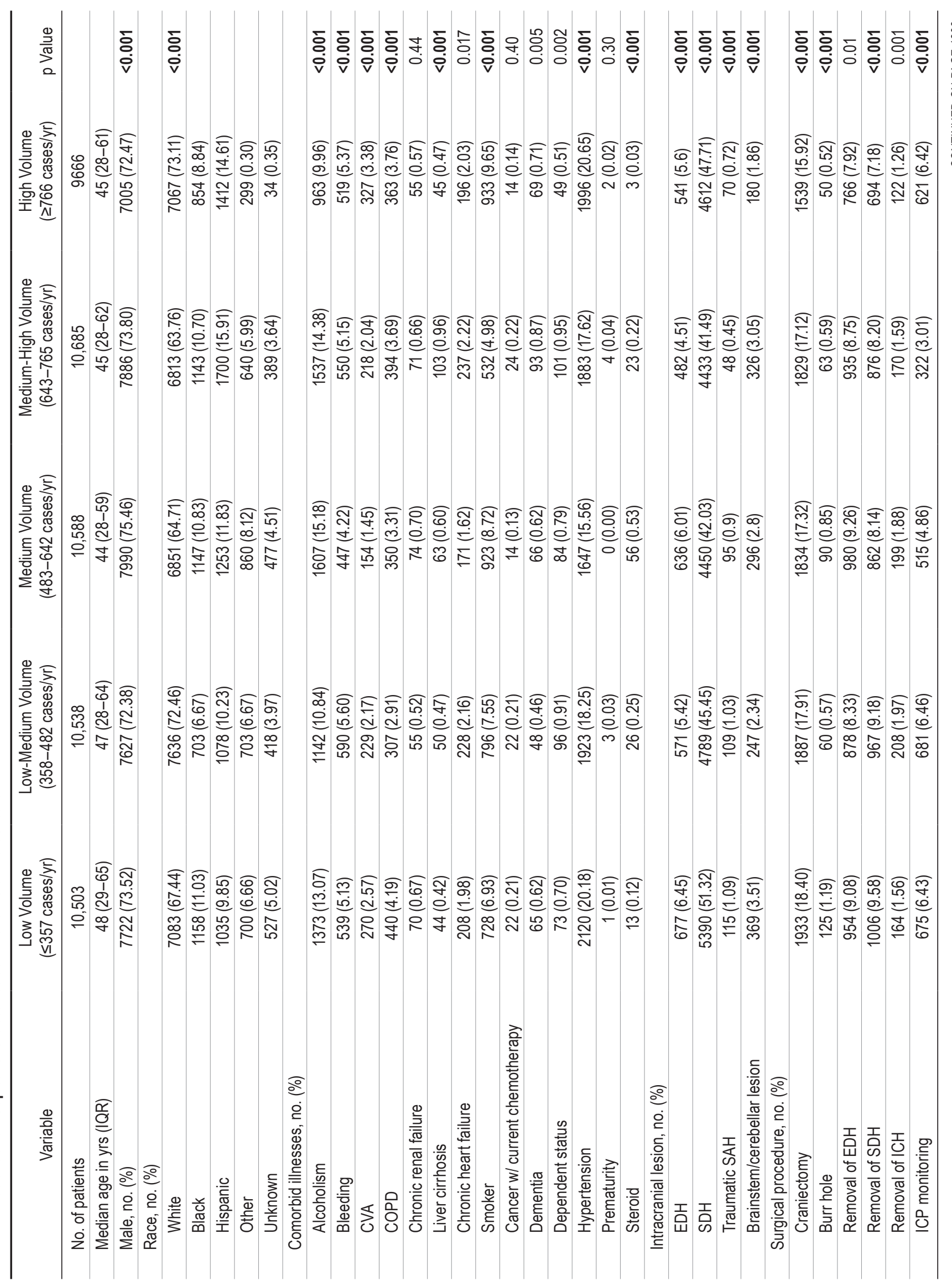




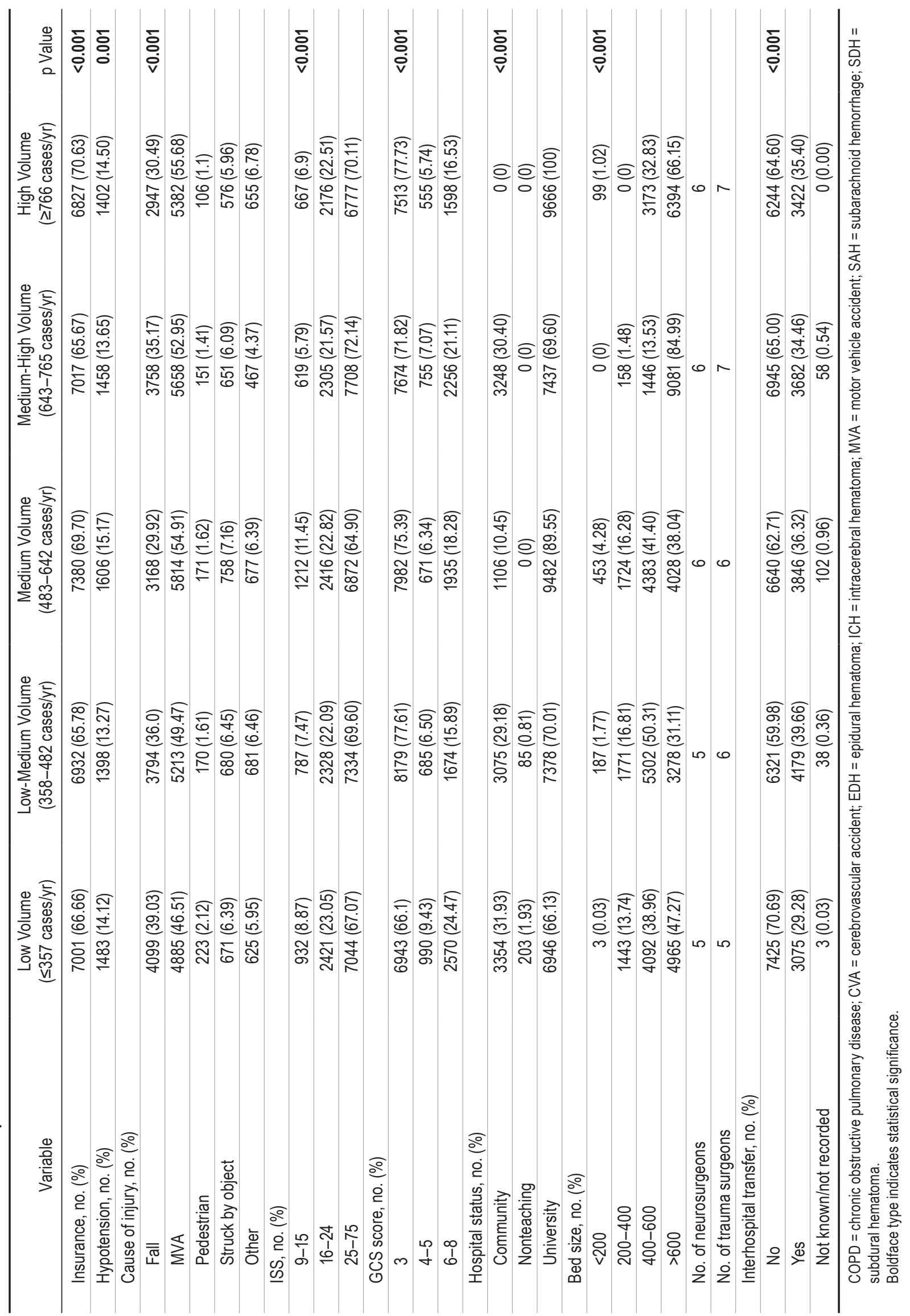




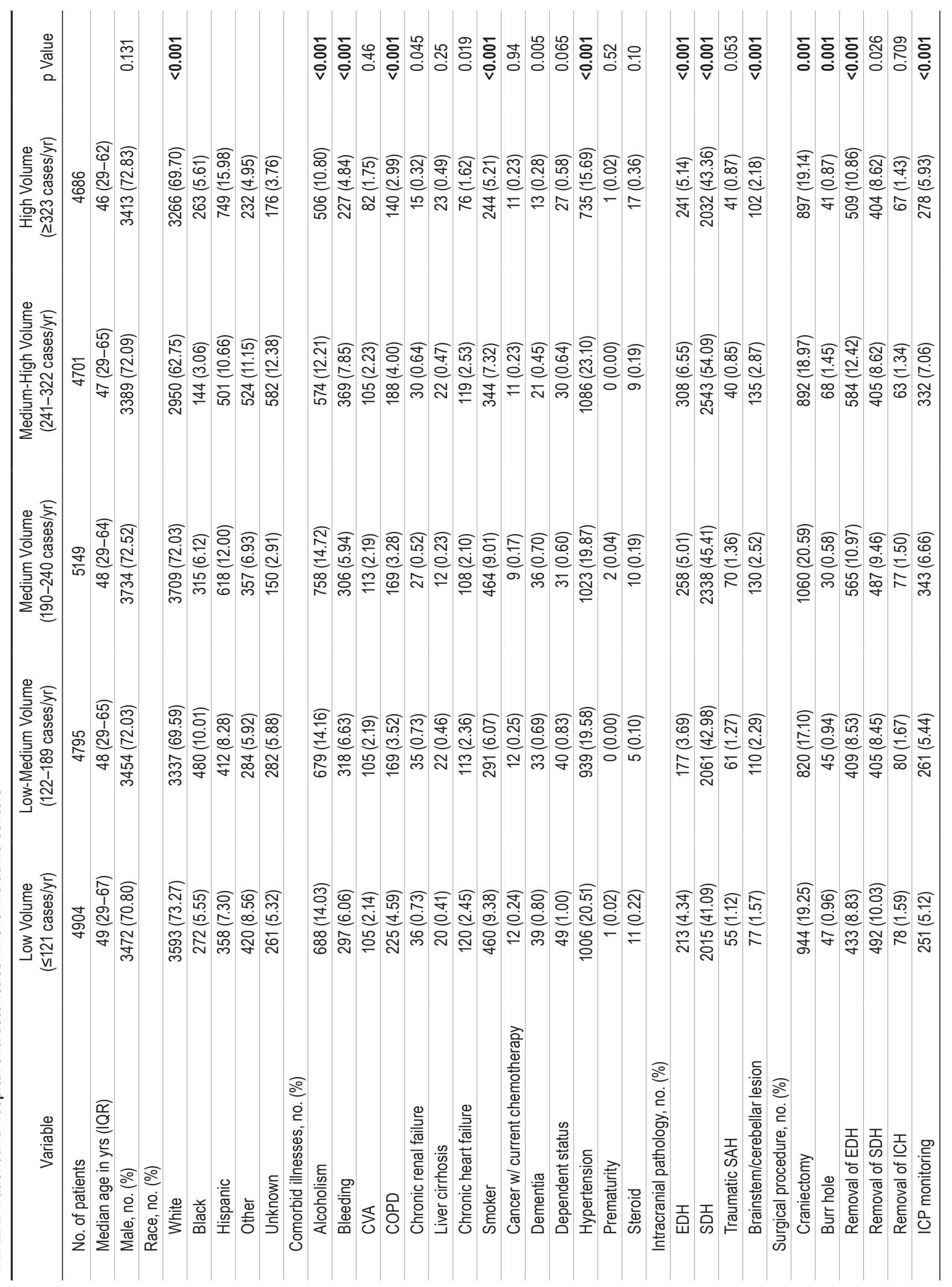




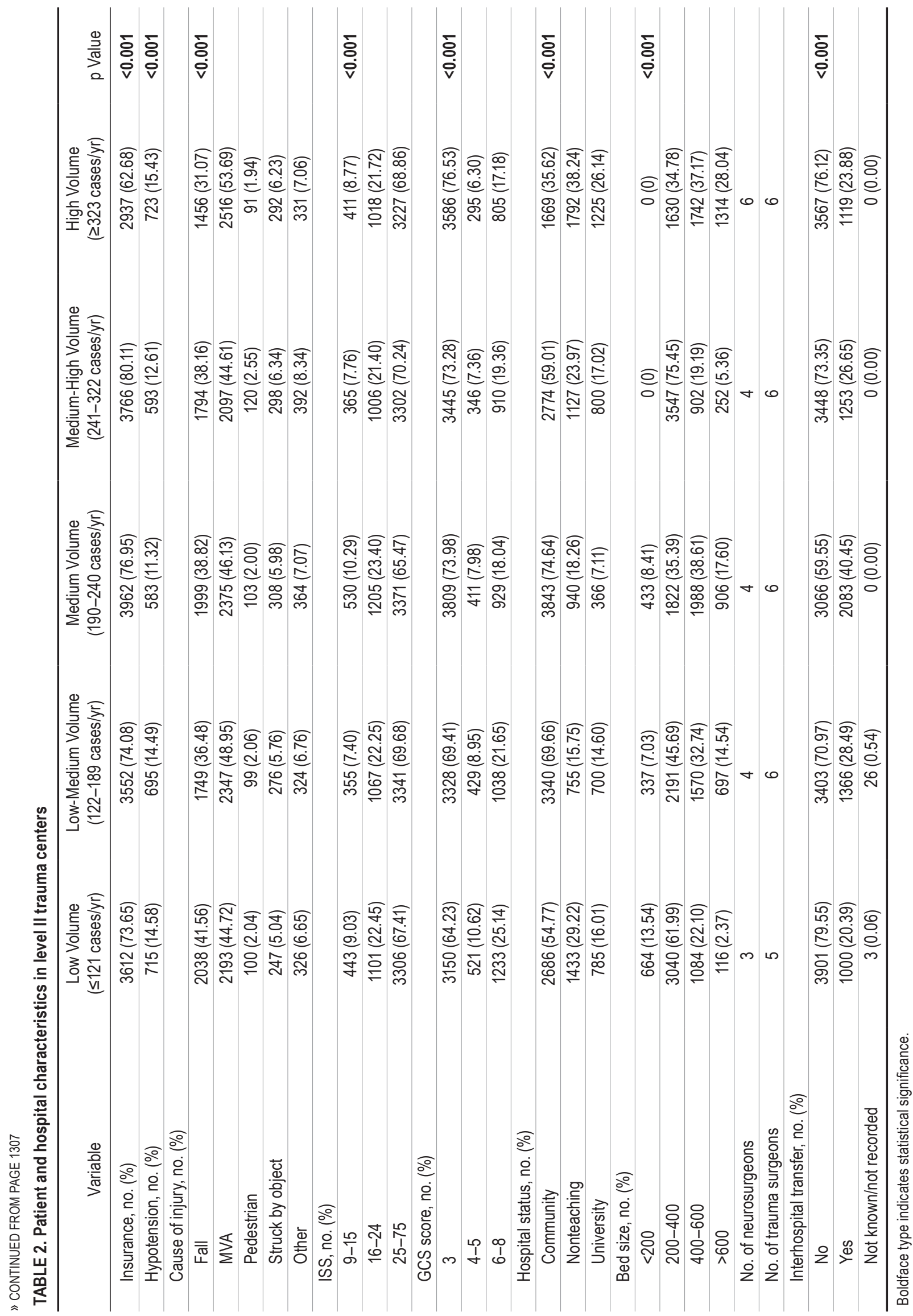


TABLE 3. Patient and hospital characteristics in level III and level IV trauma centers

\begin{tabular}{|c|c|c|c|c|c|c|}
\hline Variable & $\begin{array}{l}\text { Low Volume } \\
\text { ( } \leq 16 \text { cases/yr) }\end{array}$ & $\begin{array}{l}\text { Low-Medium } \\
\text { Volume } \\
\text { (17-21 cases/yr) }\end{array}$ & $\begin{array}{l}\text { Medium Volume } \\
\text { (22-33 cases/yr) }\end{array}$ & $\begin{array}{c}\text { Medium-High } \\
\text { Volume } \\
\text { (34-56 cases/yr) }\end{array}$ & $\begin{array}{l}\text { High Volume } \\
\text { ( } \geq 57 \text { cases/yr) }\end{array}$ & $\mathrm{p}$ Value \\
\hline No. of patients & 285 & 300 & 309 & 229 & 254 & \\
\hline Median age in yrs (IQR) & $57(39-76)$ & $54(36-74)$ & $49(29-65)$ & $47(32-71)$ & $51.5(34-73)$ & \\
\hline Male, no. (\%) & $193(67.72)$ & $203(67.67)$ & $237(76.70)$ & $152(66.38)$ & $165(64.96)$ & 0.048 \\
\hline \multicolumn{7}{|l|}{ Race, no. (\%) } \\
\hline White & $220(77.19)$ & $240(80.00)$ & $226(73.14)$ & $198(86.46)$ & $156(61.42)$ & $<0.001$ \\
\hline Black & $28(9.82)$ & $26(8.67)$ & $5(1.62)$ & $18(7.86)$ & $6(2.36)$ & \\
\hline Hispanic & $14(4.91)$ & $8(2.67)$ & $11(3.56)$ & $3(1.31)$ & $56(22.05)$ & \\
\hline Other & $5(1.75)$ & $9(3.00)$ & $10(3.24)$ & $8(3.49)$ & $10(3.94)$ & \\
\hline Unknown & $18(6.32)$ & $17(5.67)$ & $57(18.45)$ & $2(0.87)$ & $26(10.24)$ & \\
\hline \multicolumn{7}{|l|}{ Comorbid illnesses, no. (\%) } \\
\hline Alcoholism & $34(11.93)$ & $33(11.00)$ & $31(10.03)$ & $16(6.99)$ & $32(12.60)$ & 0.30 \\
\hline Bleeding & $13(4.56)$ & $14(4.67)$ & $18(5.83)$ & $16(6.99)$ & $14(5.51)$ & 0.75 \\
\hline CVA & $15(5.26)$ & $9(3.00)$ & $2(0.65)$ & $5(2.18)$ & $1(0.39)$ & $<0.001$ \\
\hline COPD & $17(5.96)$ & $13(4.33)$ & $12(3.88)$ & $11(4.80)$ & $1(0.39)$ & 0.016 \\
\hline Chronic renal failure & $1(0.35)$ & $1(0.33)$ & $0(0.00)$ & $2(0.87)$ & $1(0.39)$ & 0.59 \\
\hline Liver cirrhosis & $0(0.00)$ & $1(0.33)$ & $0(0.00)$ & $1(0.44)$ & $0(0.00)$ & 0.51 \\
\hline Chronic heart failure & $12(4.21)$ & $15(5.00)$ & $3(0.97)$ & $4(1.75)$ & $11(4.33)$ & 0.023 \\
\hline Smoker & $11(3.86)$ & $6(2.00)$ & $19(6.15)$ & $18(7.86)$ & $8(3.15)$ & 0.008 \\
\hline Cancer w/ current chemotherapy & $0(0.00)$ & $2(0.67)$ & $0(0.00)$ & $1(0.44)$ & $0(0.00)$ & 0.27 \\
\hline Dementia & $3(1.05)$ & $2(0.67)$ & $4(1.29)$ & $3(1.31)$ & $1(0.39)$ & 0.77 \\
\hline Dependent status & $4(1.40)$ & $6(2.00)$ & $3(0.97)$ & $1(0.44)$ & $1(0.39)$ & 0.32 \\
\hline Hypertension & $60(21.05)$ & $76(25.33)$ & $53(17.15)$ & $51(22.27)$ & $53(20.87)$ & 0.18 \\
\hline \multicolumn{7}{|l|}{ Intracranial lesion, no. (\%) } \\
\hline $\mathrm{EDH}$ & $13(4.56)$ & $8(2.67)$ & $18(5.83)$ & $9(3.93)$ & $7(2.76)$ & 0.254 \\
\hline $\mathrm{SDH}$ & $130(45.61)$ & $133(44.33)$ & $135(43.69)$ & $103(44.98)$ & $98(38.58)$ & 0.507 \\
\hline Traumatic SAH & $1(0.35)$ & $1(0.33)$ & $2(0.65)$ & $1(0.44)$ & $1(0.39)$ & 0.978 \\
\hline Brainstem/cerebellar lesion & $3(1.05)$ & $2(0.67)$ & $4(1.29)$ & $4(1.75)$ & $1(0.39)$ & 0.592 \\
\hline \multicolumn{7}{|l|}{ Surgical procedure, no. (\%) } \\
\hline Craniectomy & $8(2.81)$ & $22(7.33)$ & $34(11.00)$ & $12(5.24)$ & $41(16.14)$ & $<0.001$ \\
\hline Burr hole & $0(0.00)$ & $1(0.33)$ & $2(0.65)$ & $0(0.00)$ & $1(0.39)$ & 0.563 \\
\hline Removal of EDH & $2(0.70)$ & $10(3.33)$ & $16(5.18)$ & $3(1.31)$ & $30(11.81)$ & $<0.001$ \\
\hline Removal of SDH & $7(2.46)$ & $11(3.67)$ & $15(4.85)$ & $8(3.49)$ & $6(2.36)$ & 0.455 \\
\hline Removal of ICH & $1(0.35)$ & $4(1.33)$ & $3(0.97)$ & $2(0.87)$ & $8(3.15)$ & 0.054 \\
\hline ICP monitoring & $0(0.00)$ & $8(2.67)$ & $2(0.65)$ & $3(1.31)$ & $12(4.72)$ & $<0.001$ \\
\hline Insurance, no. (\%) & $222(77.89)$ & $215(71.67)$ & $186(60.19)$ & $183(79.91)$ & $209(82.28)$ & 0.002 \\
\hline Hypotension, no. (\%) & $41(14.39)$ & $33(11.0)$ & $47(15.21)$ & $30(13.10)$ & $34(13.39)$ & 0.579 \\
\hline \multicolumn{7}{|l|}{ Cause of injury, no. (\%) } \\
\hline Fall & $153(53.68)$ & $152(50.67)$ & $146(47.25)$ & $89(38.86)$ & $126(49.61)$ & $<0.001$ \\
\hline MVA & $96(33.68)$ & $109(36.33)$ & $129(41.75)$ & $101(44.10)$ & $100(39.37)$ & \\
\hline Pedestrian & $4(1.40)$ & $4(1.33)$ & $6(1.94)$ & $3(1.31)$ & $10(3.94)$ & \\
\hline Struck by object & $20(7.02)$ & $18(6)$ & $13(4.21)$ & $19(8.30)$ & $6(2.36)$ & \\
\hline Other & $12(4.21)$ & $17(5.67)$ & $15(4.85)$ & $17(7.42)$ & $12(4.72)$ & \\
\hline \multicolumn{7}{|l|}{ ISS, no. (\%) } \\
\hline $9-15$ & $45(15.79)$ & $40(13.33)$ & $51(16.50)$ & $24(10.48)$ & $3(1.18)$ & $<0.001$ \\
\hline $16-24$ & $81(28.42)$ & $85(28.33)$ & 77 (24.92) & $55(24.02)$ & $57(22.44)$ & \\
\hline $25-75$ & $154(54.04)$ & $170(56.67)$ & $173(55.99)$ & $147(64.19)$ & $192(75.59)$ & \\
\hline
\end{tabular}


TABLE 3. Patient and hospital characteristics in level III and level IV trauma centers

\begin{tabular}{|c|c|c|c|c|c|c|}
\hline Variable & $\begin{array}{l}\text { Low Volume } \\
\text { ( } \leq 16 \text { cases/yr) }\end{array}$ & $\begin{array}{c}\text { Low-Medium } \\
\text { Volume } \\
\text { (17-21 cases/yr) }\end{array}$ & $\begin{array}{l}\text { Medium Volume } \\
\text { (22-33 cases/yr) }\end{array}$ & $\begin{array}{c}\text { Medium-High } \\
\text { Volume } \\
\text { (34-56 cases/yr) }\end{array}$ & $\begin{array}{l}\text { High Volume } \\
\text { ( } \geq 57 \text { cases/yr) }\end{array}$ & $p$ Value \\
\hline \multicolumn{7}{|l|}{ GCS score, no. (\%) } \\
\hline 3 & $146(51.23)$ & $179(59.67)$ & $173(55.99)$ & $134(58.52)$ & $163(64.17)$ & $<0.001$ \\
\hline $4-5$ & $37(12.98)$ & $38(12.67)$ & $39(12.62)$ & $20(8.73)$ & $34(13.39)$ & \\
\hline $6-8$ & $102(35.79)$ & $83(27.67)$ & $97(31.39)$ & $75(32.75)$ & $57(22.44)$ & \\
\hline \multicolumn{7}{|l|}{ Hospital status, no. (\%) } \\
\hline Community & $82(28.77)$ & $81(27.00)$ & $96(31.07)$ & $95(41.48)$ & $73(28.74)$ & $<0.001$ \\
\hline Nonteaching & $153(53.68)$ & $190(63.33)$ & $124(40.13)$ & $105(45.85)$ & $159(62.60)$ & \\
\hline University & $17(5.96)$ & $28(9.33)$ & $0(0)$ & $28(12.23)$ & $0(0)$ & \\
\hline Unknown & $33(11.58)$ & $1(0.33)$ & $89(28.80)$ & $1(0.44)$ & $22(8.66)$ & \\
\hline \multicolumn{7}{|l|}{ Bed size, no. (\%) } \\
\hline$<200$ & $149(52.28)$ & $183(61)$ & $138(44.66)$ & $49(21.40)$ & $16(6.30)$ & $<0.001$ \\
\hline $200-400$ & $109(38.25)$ & $69(23)$ & $112(36.25)$ & $180(78.60)$ & $194(76.38)$ & \\
\hline $400-600$ & $21(7.37)$ & $48(16)$ & $59(19.09)$ & $0(0)$ & $30(11.81)$ & \\
\hline$>600$ & $6(2.11)$ & $0(0)$ & $0(0)$ & $0(0)$ & $14(5.51)$ & \\
\hline No. of neurosurgeons & 0 & 0 & 0 & 1 & 2 & \\
\hline No. of trauma surgeons & 4 & 4 & 4 & 5 & 9 & \\
\hline Interhospital transfer, no. (\%) & & & & & & $<0.001$ \\
\hline No & $273(95.79)$ & $287(95.67)$ & $291(94.17)$ & $208(90.83)$ & $211(83.07)$ & \\
\hline Yes & $11(3.86)$ & $13(4.33)$ & $18(5.83)$ & $21(9.17)$ & $43(16.93)$ & \\
\hline Not known/not recorded & $1(0.35)$ & $0(0.00)$ & $0(0.00)$ & $0(0.00)$ & $0(0.00)$ & \\
\hline
\end{tabular}

Boldface type indicates statistical significance.

centers (77\%) than in low-volume centers (66\%). In level III and level IV trauma centers, case volume quintiles were defined as $\leq 16,17-21,22-33,34-56$, and $\geq 57$ cases/year, respectively. Table 3 outlines baseline patient- and hospital-level characteristics for level III and IV trauma centers by volume quintile.

\section{Outcomes}

Among level I trauma centers, adjusted mortality and complication rates, as well as total hospital and ICU stays, did not reveal significant differences with each stratum of increasing hospital volume (Table 4). Similarly, among level II trauma centers, a significant volume-outcome relationship was not observed for mortality, complications, or hospital discharge disposition (Table 5). However, unadjusted analysis showed total LOS was longer in highervolume facilities compared to the lowest-volume facilities (unadjusted MD: Q2, 1.97 days, $\mathrm{p}<0.001 ; \mathrm{Q} 3,2.16$ days, $\mathrm{p}$ $<0.001 ; \mathrm{Q} 4,1.58$ days, $\mathrm{p}=0.03$; $\mathrm{Q} 5,3.33$ days, $\mathrm{p}<0.001$; reference $=\mathrm{Q} 1)$. On adjusted analysis, $\mathrm{LOS}$ was significantly longer with the highest-volume stratum (Q5) compared to low-volume facilities (adjusted MD: Q5, 2.83 days, 95\% CI 1.4-4.3 days, $\mathrm{p}<0.001$, reference $=\mathrm{Q} 1)$. Similarly, unadjusted ICU stay was also longer in higher-volume facilities than in the lowest-volume facilities (unadjusted MD: $\mathrm{Q} 2,1.26$ days, $\mathrm{p}<0.001 ; \mathrm{Q} 3,1.24$ days, $\mathrm{p}<0.001 ; \mathrm{Q} 4,0.84$ days, $\mathrm{p}=0.07 ; \mathrm{Q} 5,1.6$ days, $\mathrm{p}<0.001)$. However, adjusted analysis did not reveal a significant difference in ICU stay with increasing volume quintile (adjusted MD: Q2, 1.13 days, $95 \%$ CI $0.41-1.85, \mathrm{p}=0.002$; $\mathrm{Q} 3,1.12$ days, $95 \% \mathrm{CI}$ $0.42-2.00, \mathrm{p}=0.003 ; \mathrm{Q} 4,0.64$ days, $95 \% \mathrm{CI}-0.25$ to 1.54 , $\mathrm{p}=0.16$; $\mathrm{Q} 5,1.32$ days, 95\% CI 0.38-2.26, $\mathrm{p}=0.006$ ).

For level III and level IV trauma centers (Table 6), the adjusted odds ratios suggested greater mortality with each quintile of severe TBI hospital volume but the relationships were statistically nonsignificant (low-medium volume: OR $1.71,95 \%$ CI $0.66-4.38, p=0.27$; medium volume: OR 2.81, 95\% CI 0.93-8.47, $\mathrm{p}=0.07$; mediumhigh volume: OR 1.18, 95\% CI 0.30-4.57, $\mathrm{p}=0.81$; high volume: OR $2.48,95 \%$ CI $0.68-9.08, \mathrm{p}=0.17$; reference $=$ low volume). Similarly, complication rates were also higher with increasing case volume but the relationships were statistically nonsignificant on adjusted analysis (low-medium volume: OR 2.39, 95\% CI 0.73-7.87, $\mathrm{p}=0.15$; medium volume: OR $0.64,95 \%$ CI $0.14-2.96, \mathrm{p}=0.57$; mediumhigh volume: OR 2.87, 95\% CI 0.54-15.20, $\mathrm{p}=0.21$; high volume: OR 3.92, 95\% CI 0.70-21.85, $\mathrm{p}=0.12$; reference $=$ low volume). Similarly, we found higher odds of nonhome discharge with higher-volume quintiles; however, these relationships were not statistically significant (low-medium volume: OR $1.63,95 \%$ CI $0.50-5.35, \mathrm{p}=0.42$; medium volume: OR 2.16, 95\% CI 0.55-8.47, $\mathrm{p}=0.27$; mediumhigh volume: OR 3.34, 95\% CI 0.89-12.43, $\mathrm{p}=0.07$; high volume: OR $1.16,95 \%$ CI 0.19-7.21, $\mathrm{p}=0.87$; reference $=$ low volume). Adjusted hospital LOS was approximately 5 days longer in the highest-volume stratum ( $\geq 57$ cases/year, 


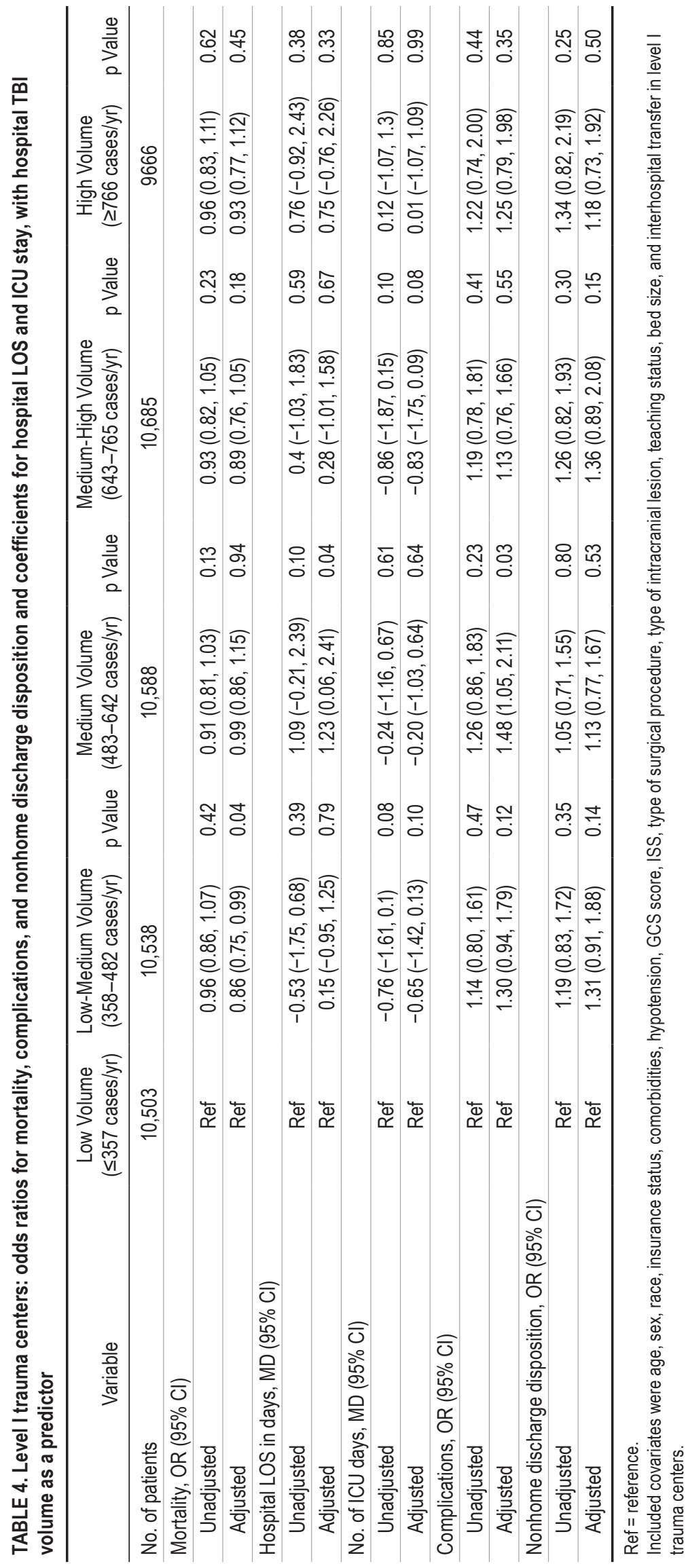




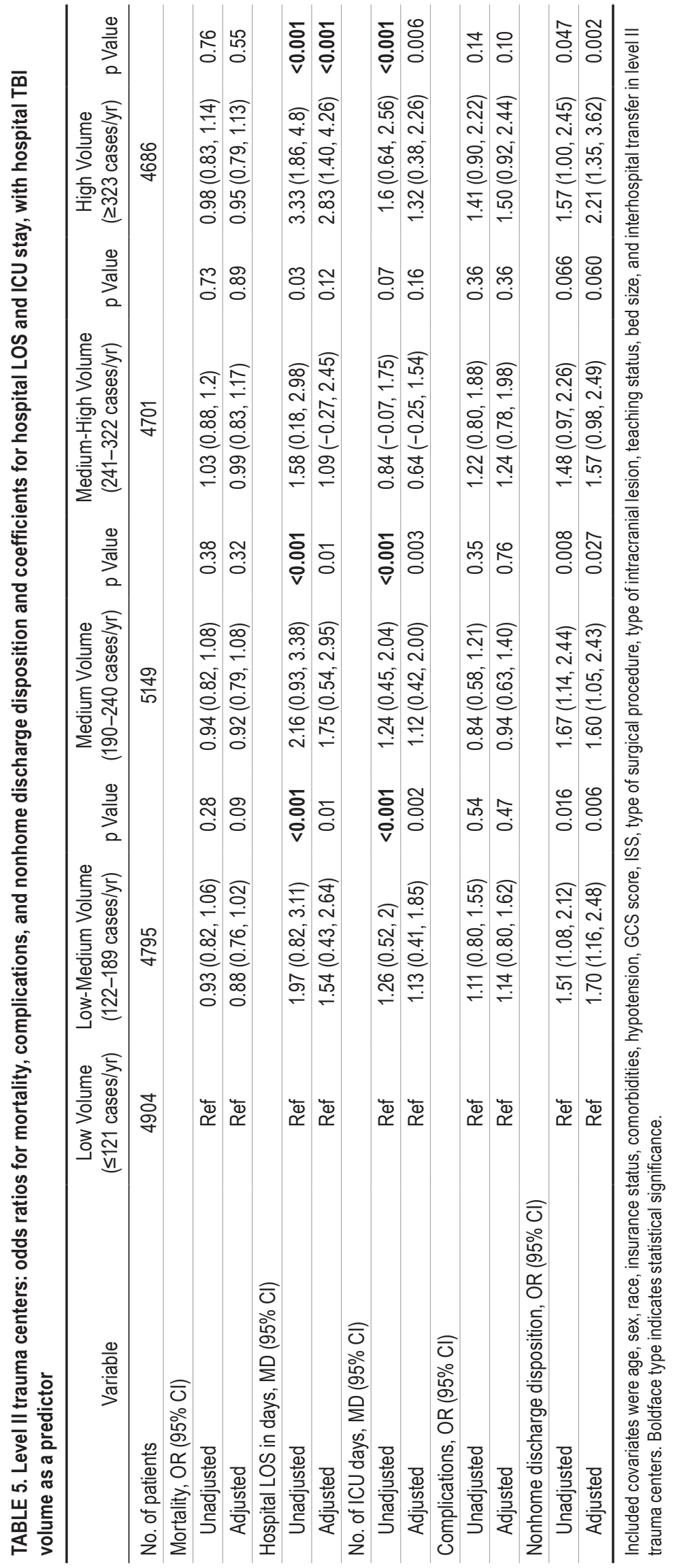




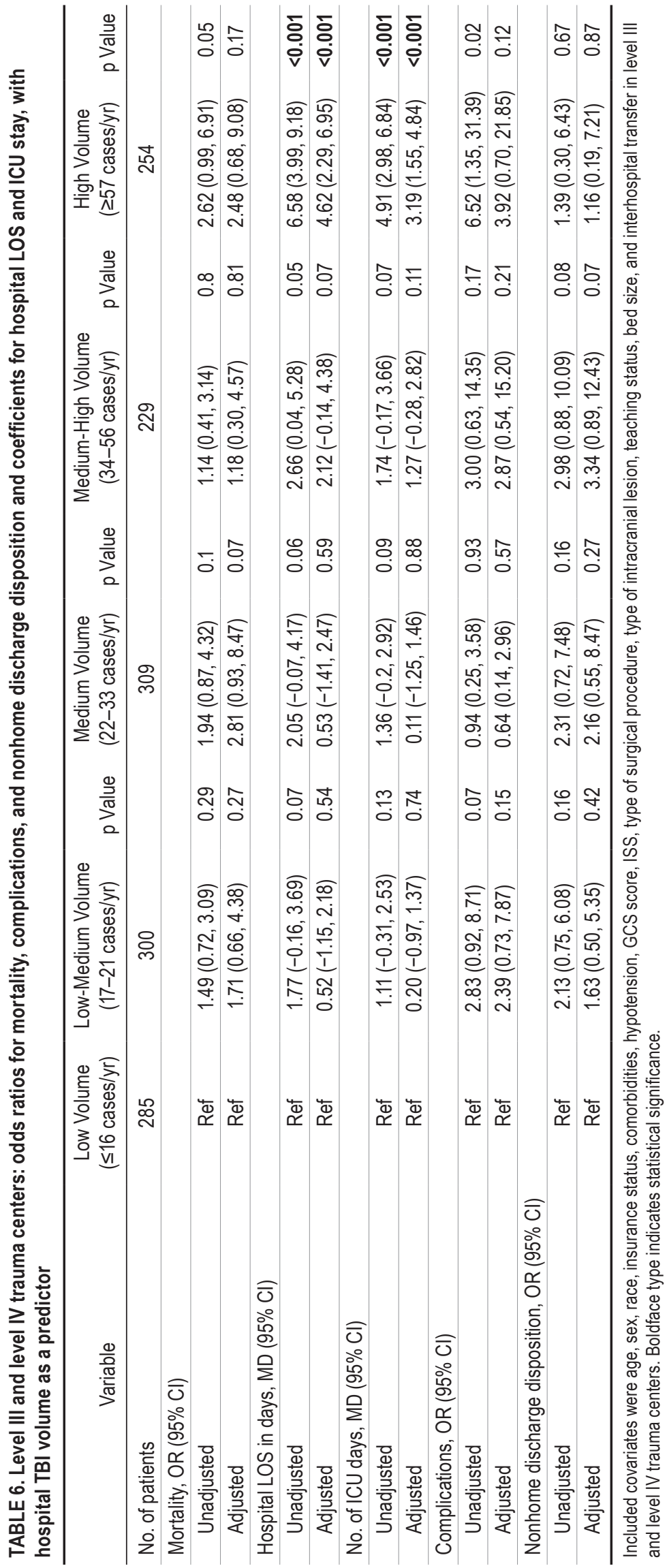


$\mathrm{p}<0.001)$. Adjusted ICU stay was approximately 3 days longer in the highest-volume stratum $(\mathrm{p}<0.001)$.

\section{Discussion}

The aim of this study was to determine the relationship between case volume and in-hospital mortality, in-hospital complications, hospital LOS, and ICU stay in patients with severe TBI. Most other studies focusing on the hospital volume-outcome relationship have not studied the effect of the level designation of trauma centers and instead have pooled various levels of trauma centers in their analyses. ${ }^{1,20,21}$ In our investigation, however, we distinguished the effect of the level designation to see whether the volume stratum at a particular designation had an effect on patient outcome. Our study did not reveal a significant volume-outcome relationship for in-hospital mortality, complications, total hospital stay, and ICU stay among level I trauma centers. These results corroborate findings in an observational study by Wada et al., who did not find hospital TBI volume to be significantly associated with mortality in an analysis of a trauma database in Japan. ${ }^{21}$ However, we did observe an increase in adverse outcomes with increasing volume among level II, level III, and level IV trauma centers. Patients treated at level II, III, and IV trauma centers tended to stay longer in higher-volume facilities compared to patients in lower-volume centers, which is contradictory to findings in the literature. ${ }^{19}$ The effect size (odds ratios and confidence intervals) for higher mortality and complications with increasing volume at these centers was inconclusive, suggesting more studies are needed to investigate this relationship for level III and IV centers.

These results probably indicate that a higher annual severe TBI volume at a lower level of trauma center may be associated with adverse outcomes. Our study design was similar to a study conducted by Alali et al., which used data from the ACS Trauma Quality Improvement Program. ${ }^{1}$ Their study included 111 level I and level II trauma centers that participated in the program during 2009-2011. ${ }^{1}$ We included subjects enrolled over a longer period (2007-2014) in the NTDB, with a larger sample size. In addition, we performed a stratified analysis by level of trauma center since our hypothesis was that the effect of severe TBI hospital volume on outcomes would be different according to the type of hospital given the differences in resources available for trauma care. Since there are other criteria that determine the level of trauma center and associated outcomes, such as educational programs, resource allocation, and injury prevention, there may be other factors associated with outcomes in various levels of trauma centers rather than volume alone. ${ }^{2}$ Therefore, our results demonstrate the complexity in improving the outcomes at trauma centers, which are probably influenced by factors other than volume itself. Glance et al. performed an NTDB study of 7371 patients with ISS $\geq 15$ and also failed to demonstrate any association between trauma volume and in-hospital outcomes for both blunt and penetrating trauma. ${ }^{6}$

In our study, we only included subjects with an age $\geq 18$ years, GCS score $\leq 8$, and AIS head score $\geq 3$. We excluded cases with penetrating trauma because most of these cases would be transferred to a level I or level II trauma center and because these cases usually have worse outcomes than those in blunt trauma. Unlike previous studies, which have relied on ICD-9 and ICD-10 diagnosis codes, our inclusion definition of severe TBI used the GCS and ISS, thereby increasing the strength of our findings. We restricted our investigation to severe TBI cases defined as a GCS score $\leq$ 8 and AIS head score $>2$. We also performed adjustment for the type of intracranial pathology (subdural hematoma, epidural hematoma, traumatic subarachnoid hemorrhage, and cerebellar/brainstem lesions) and the surgical intervention performed.

\section{Study Strengths and Limitations}

The strengths of our study include a large sample size and adjustment for several patient-level factors such as insurance status, surgical procedure, case severity, type of intracranial pathology, and hospital-level characteristics. Using a clinical definition in place of administrative ICD9 codes to identify severe TBI reduced the risk of selection bias due to inaccurate diagnosis. We used a stringent definition for statistical significance to avoid spurious associations.

However, there are limitations to this study. Despite adjustment for various confounders, there is a possibility of additional residual confounding, which could have affected our results. National databases allow pooling of a large number of cases, but there is limited granularity in the amount of clinically relevant variables available for adjustment. For instance, withdrawal-of-care decisions are not explicitly captured in the database. However, the number of cases from level III and level IV trauma centers was significantly smaller than those of level I and level II trauma centers, which could limit our ability to draw conclusions about in-hospital mortality at these centers. Second, inferences can only be made about associations, and there is limited evidence to support causality given the observational nature of our study. Third, residual unmeasured confounding that was not adjusted for in the current analysis may have influenced the results. For instance, information about rural versus urban hospital location could not be gathered, which could have an impact on transport to level I trauma centers from level III or level IV centers. Future investigations should also consider examining whether a change in volume with time at a given trauma center would impact outcomes.

\section{Conclusions}

Analysis based on the ACS NTDB did not reveal a significant volume-outcome relationship for severe TBI in level I trauma centers. However, we found that a higher volume of severe TBI in lower levels of trauma centers may be associated with longer LOSs. This finding may suggest a need for improved resource allocation for comparatively ill-equipped trauma centers, careful prehospital decision-making, and timely referral of patients with severe injury to a higher level of care, to improve outcomes in patients with TBI.

\section{References}

1. Alali AS, Gomez D, McCredie V, Mainprize TG, Nathens AB: Understanding hospital volume-outcome relationship in 
severe traumatic brain injury. Neurosurgery 80:534-542, 2017

2. American College of Surgeons: Resources for Optimal Care of the Injured Patient 2014, ed 6. Chicago: ACS, 2014 (https://www.facs.org/-/media/files/quality-programs/trauma/ vrc-resources/resources-for-optimal-care.ashx) [Accessed January 25, 2020]

3. Caputo LM, Salottolo KM, Slone DS, Mains CW, Bar-Or D: The relationship between patient volume and mortality in American trauma centres: a systematic review of the evidence. Injury 45:478-486, 2014

4. Clark DE, Hannan EL, Wu C: Predicting risk-adjusted mortality for trauma patients: logistic versus multilevel logistic models. J Am Coll Surg 211:224-231, 2010

5. Giwa F, Salami A, Abioye AI: Hospital esophagectomy volume and postoperative length of stay: a systematic review and meta-analysis. Am J Surg 215:155-162, 2018

6. Glance LG, Osler TM, Dick A, Mukamel D: The relation between trauma center outcome and volume in the National Trauma Databank. J Trauma 56:682-690, 2004

7. Goka EA, Phillips P, Poku E, Essat M, Woods HB, Walters SJ, et al: The relationship between hospital or surgeon volume and outcomes in lower limb vascular surgery in the United Kingdom and Europe. Ann Vasc Surg 45:271-286, 2017

8. Haider AH, Saleem T, Leow JJ, Villegas CV, Kisat M, Schneider EB, et al: Influence of the National Trauma Data Bank on the study of trauma outcomes: is it time to set research best practices to further enhance its impact? J Am Coll Surg 214:756-768, 2012

9. Hata T, Motoi F, Ishida M, Naitoh T, Katayose Y, Egawa S, et al: Effect of hospital volume on surgical outcomes after pancreaticoduodenectomy: a systematic review and metaanalysis. Ann Surg 263:664-672, 2016

10. Hsu RCJ, Salika T, Maw J, Lyratzopoulos G, Gnanapragasam VJ, Armitage JN: Influence of hospital volume on nephrectomy mortality and complications: a systematic review and meta-analysis stratified by surgical type. BMJ Open 7:e016833, 2017

11. Kim YJ: Relationship of trauma centre characteristics and patient outcomes: a systematic review. J Clin Nurs 23:301314, 2014

12. Leow JJ, Leong EK, Serrell EC, Chang SL, Gruen RL, Png $\mathrm{KS}$, et al: Systematic review of the volume-outcome relationship for radical prostatectomy. Eur Urol Focus 4:775-789, 2018

13. London JA, Battistella FD: Is there a relationship between trauma center volume and mortality? J Trauma 54:16-25, 2003

14. Melfa G, Porello C, Cocorullo G, Raspanti C, Rotolo G, Attard A, et al: Surgeon volume and hospital volume in endocrine neck surgery: how many procedures are needed for reaching a safety level and acceptable costs? A systematic narrative review. G Chir 39:5-11, 2018

15. Minei JP, Fabian TC, Guffey DM, Newgard CD, Bulger EM, Brasel KJ, et al: Increased trauma center volume is associated with improved survival after severe injury: results of a Resuscitation Outcomes Consortium study. Ann Surg 260:456-465, 2014
16. Panaich SS, Patel N, Arora S, Patel NJ, Patel SV, Savani C, et al: Influence of hospital volume and outcomes of adult structural heart procedures. World J Cardiol 8:302-309, 2016

17. Phillips P, Poku E, Essat M, Woods HB, Goka EA, Kaltenthaler EC, et al: Procedure volume and the association with short-term mortality following abdominal aortic aneurysm repair in European populations: a systematic review. Eur J Vasc Endovasc Surg 53:77-88, 2017

18. Preston L, Turner J, Booth A, O'Keeffe C, Campbell F, Jesurasa $\mathrm{A}$, et al: Is there a relationship between surgical case volume and mortality in congenital heart disease services? A rapid evidence review. BMJ Open 5:e009252, 2015

19. Shi HY, Hwang SL, Lee IC, Chen IT, Lee KT, Lin CL: Trends and outcome predictors after traumatic brain injury surgery: a nationwide population-based study in Taiwan. J Neurosurg 121:1323, 2014

20. Tepas JJ III, Pracht EE, Orban BL, Flint LM: High-volume trauma centers have better outcomes treating traumatic brain injury. J Trauma Acute Care Surg 74:143-148, 2013

21. Wada T, Yasunaga H, Doi K, Matsui H, Fushimi K, Kitsuta Y, et al: Relationship between hospital volume and outcomes in patients with traumatic brain injury: a retrospective observational study using a national inpatient database in Japan. Injury 48:1423-1431, 2017

22. Weinheimer KT, Smuin DM, Dhawan A: Patient outcomes as a function of shoulder surgeon volume: a systematic review. Arthroscopy 33:1273-1281, 2017

\section{Disclosures}

The authors report no conflict of interest concerning the materials or methods used in this study or the findings specified in this paper.

\section{Author Contributions}

Conception and design: Tsai, Goyal. Acquisition of data: Tsai, Goyal. Analysis and interpretation of data: Tsai, Goyal. Drafting the article: Tsai, Goyal. Critically revising the article: all authors. Reviewed submitted version of manuscript: all authors. Approved the final version of the manuscript on behalf of all authors: Bydon. Statistical analysis: Tsai, Goyal, Wahood. Administrative/ technical/material support: Bydon, Habermann. Study supervision: Bydon.

\section{Supplemental Information \\ Online-Only Content}

Supplemental material is available with the online version of the article.

Supplementary Table 1. https://thejns.org/doi/suppl/10.3171/ 2020.1.JNS192115.

\section{Correspondence}

Mohamad Bydon: Mayo Clinic, Rochester, MN. bydon. mohamad@mayo.edu. 